\title{
Bloodstream infection in patients with end-stage renal disease in a teaching hospital in central-western Brazil
}

\author{
Tamara Trelha Gauna ${ }^{[1]}$, Elizete Oshiro ${ }^{[2]}$, Yuri Correa Luzio ${ }^{[2]}$, \\ Anamaria Mello Miranda Paniago ${ }^{[2]}$, Elenir Rose Jardim Cury Pontes ${ }^{[3]}$ \\ and Marilene Rodrigues Chang ${ }^{[4]}$
}

[1]. Hospital Universitário Maria Aparecida Pedrossian, Universidade Federal de Mato Groso do Sul, Campo Grande, MS. [2]. Faculdade de Medicina, Universidade Federal de Mato Grosso do Sul, Campo Grande, MS. [3]. Departamento de Tecnologia de Alimentos e Saúde Pública, Universidade Federal de Mato Grosso do Sul, Campo Grande, MS. [4]. Departamento de Farmácia, Universidade Federal de Mato Grosso do Sul, Campo Grande, MS.

\begin{abstract}
Introduction: Vascular access in patients undergoing hemodialysis is considered a critical determinant of bloodstream infection (BSI) and is associated with high morbidity and mortality. The purpose of this study was to investigate the occurrence of BSI in patients with end-stage renal disease using central venous catheters for hemodialysis. Methods: A cohort study was conducted in a public teaching hospital in central-western Brazil from April 2010 to December 2011. For every patient, we noted the presence of hyperemia/exudation upon catheter insertion, as well as fever, shivering, and chills during hemodialysis. Results: Fifty-nine patients were evaluated. Thirty-five (59.3\%) patients started dialysis due to urgency, 37 (62.7\%) had BSI, and $12(20 \%)$ died. Hyperemia at the catheter insertion site (64.9\%) was a significant clinical manifestation in patients with BSI. Statistical analysis revealed 1.7 times more cases of BSI in patients with hypoalbuminemia compared with patients with normal albumin levels. The principal infective agents identified in blood cultures and catheter-tip cultures were Staphylococcus species (24 cases), non-fermentative Gram-negative bacilli (7 cases of Stenotrophomonas maltophilia and 5 cases of Chryseobacterium indologenes), and Candida species (6). Among the Staphylococci identified, 77.7\% were methicillin-resistant, coagulase-negative Staphylococci. Of the bacteria isolated, the most resistant were Chryseobacterium indologenes and Acinetobacter baumannii. Conclusions: Blood culture was demonstrated to be an important diagnostic test and identified over $50 \%$ of positive BSI cases. The high frequency of BSI and the isolation of multiresistant bacteria were disturbing findings. Staphylococcus aureus was the most frequently isolated microorganism, although Gram-negative bacteria predominated overall. These results highlight the importance of infection prevention and control measures in dialysis units.
\end{abstract}

Keywords: Catheter. Bloodstream infection. Chronic renal patients. Bacteremia. Hemodialysis.

\section{INTRODUCTION}

End-stage renal disease (ESRD) is considered a global public health problem ${ }^{1}$. In Brazil, the number of patients with ESRD undergoing renal replacement therapy increased from 42,695 in 2000 to 92,091 in 2010 . As many as $90.6 \%$ of patients with ESRD receive hemodialysis, and $13.6 \%$ of these patients receive non-tunneled catheters ${ }^{2}$. The survival and quality of life of these patients depend on the continued good functioning of dialysis access sites ${ }^{3}$. Bloodstream infection (BSI) is the leading cause of hospitalization and the second most common cause of death among patients receiving regular hemodialysis ${ }^{4,5}$.

Address to: Dr $\stackrel{a}{=}$ Tamara Trelha Gauna. Rua Ponte Preta 55, Bairro Santo Amaro, 79112-460 Campo Grande, MS, Brasil.

Phone: 55 67 3345-3119; Cell phone: 5567 9158-7066; Fax: 5557 3345-3153 e-mail: tamaratrelha@ig.com.br

Received 17 March 2013

Accepted 10 July 2013
Controlling infection in these patients is a challenge for healthcare staff because hemodialysis is an invasive procedure with an inherent infection risk. Furthermore, catheters are often manipulated during hemodialysis sessions, and patients receiving hemodialysis are immunodeficient ${ }^{6}$.

Primary BSIs are among the most common nosocomial infections. Data suggest that $60 \%$ of hospital-acquired bacteremia cases are associated with the use of central venous catheters $(\mathrm{CVCs})^{7}$. Metastatic sites of infection occur frequently and can include endocarditis, vertebral osteomyelitis, spinal epidural abscess, and septic arthritis ${ }^{8}$. Despite the importance of BSIs, few studies have investigated this issue in patients with ESRD in Brazil. Instead, they have mostly focused on microbiological laboratory surveillance, without association of the results with clinical data ${ }^{9,10}$.

The isolation of the etiologic agent and determination of antimicrobial susceptibility profile are important for achieving better prognoses. The emergence of multiresistant bacteria is a well-recognized problem. Therefore, surveillance studies are important for the monitoring of the emergence of these microorganisms, especially in immunocompromised patients, such as those undergoing hemodialysis. 
The purpose of this study was to describe BSI in patients with ESRD using CVCs, describe the patients' characteristics, identify the etiologic agents, and analyze the catheter-related variables considered as risk factors for BSI.

\section{METHODS}

\section{Study design}

This paper reports on a prospective cohort study of 59 patients with ESRD receiving hemodialysis by means of a CVC at a Brazilian tertiary care teaching hospital in the State of Mato Grosso do Sul from April 2010 to December 2011. The principal outcome investigated was BSI.

\section{Data collection and BSI surveillance}

Included in the study were patients with ESRD using catheters and recently admitted to the hospital's hemodialysis ward, patients undergoing hemodialysis who lost an arteriovenous fistula (AVF) or a catheter, and patients with implanted tunneled or non-tunneled catheters. The sample included both ESRD inpatients and outpatients. All subjects included in the study were adults and agreed to participate. Individuals in intensivecare units and children were excluded.

Patients were followed up until four months postcatheterization. All CVCs were inserted using aseptic techniques according to the protocol recommended by the Hospital Infection Control Committee.

Data were collected during the hemodialysis sessions. We collected at least two peripheral blood samples from all patients who had signs and symptoms of BSI. Blood was collected in compliance with the criteria of the National Agency of Sanitary Surveillance ${ }^{11}$. The catheter insertion sites were inspected, and blood and/or catheter-tip cultures were monitored. Nursing staff trained in hemodialysis changed the dressings and manipulated the catheters using rigorous aseptic techniques. At the end of each hemodialysis session, the catheter lumens were aseptically locked with heparin. The catheters were not filled with any other thrombolytic agent or antimicrobial solution, and the connectors were not part of a closed access system.

For every patient, we checked for the presence of hyperemia at the catheter site, exudation upon catheter insertion, and symptoms such as fever, shivering, and chills during each hemodialysis session. Demographic, clinical, and laboratory data were collected from medical records and from the hospital's laboratory database.

Empirical systemic antibiotic therapy was initiated whenever the patients developed fever or shivering. Vancomycin and ceftazidime were the first choices for the initiated antibiotic regimen. The regimen was adjusted, if necessary, once the results of blood culture tests became available. The catheter was removed if fever or shivering symptoms persisted for more than $48 \mathrm{~h}$. In cases suggestive of BSI, according to medical criteria, the catheter tip was removed and subjected to culture. One week after antibiotic therapy initiation, new blood cultures were collected.

\section{Microbiological tests}

Blood cultures were performed in the hospital's microbiology laboratory. The laboratory used a Bactec automated system (Becton Dickinson, Sparks, USA) for blood culture tests and a Vitek2 Compact automated system (BioMérieux, Durham, NC, USA) for the identification of pathogens and antimicrobial susceptibility profiling of blood samples. In cases of blood cultures with the same microorganism for one month or consecutive months, only one agent was considered.

\section{Definitions}

Bacteremia and candidemia were defined as clinical signs or symptoms of infection along with at least two blood cultures that tested positive for bacteria or yeasts of the genus Candida ${ }^{12}$.

Diagnosis of CVC-related BSI was defined as at least one blood culture and catheter tip culture with the same agent, with clinical manifestations of infection and no other apparent source of infection ${ }^{13}$.

Bacteria were considered multidrug-resistant when exhibiting resistance to different classes of antimicrobial medications ${ }^{7}$.

Hypoalbuminemia was defined as serum albumin levels of less than $3.5 \mathrm{~g} / \mathrm{dL}^{4}$.

\section{Statistical analysis}

To investigate the possible associations between the study variables, chi-square tests, chi-square tests for trends, and Fisher's exact test were applied, and the relative risks were calculated with $95 \%$ confidence intervals. Cox regression analysis was used to estimate the adjusted relative risks using variables with significance levels lower than $20 \%$ and those of clinical and epidemiological relevance. BioEstat 5.0 (Sociedade Mamirauá, Belém, Brazil) and Epi Info (version 3.5.3) were used for the statistical analyses ${ }^{14,15}$. Statistical significance was defined as $\mathrm{p} \leq 0.05$.

\section{Ethical considerations}

Our study was approved by the Research Ethics Committee of the Federal University of Mato Grosso do Sul (permit 1687/2010). All individuals who agreed to participate in the study signed informed consent forms stating that they were freely participating.

\section{RESULTS}

Of the 59 patients with ESRD using CVCs for hemodialysis, $30(50.8 \%)$ developed bacteremia, 7 (11.6\%) had mixed bacteremia and fungemia, and $22(37.3 \%)$ had no evidence of BSI. The overall infection rate was $62.8 \%(95 \% \mathrm{CI}$, 50.4-75.1\%).

The only patient who used a tunneled catheter developed BSI. Among the other 58 patients who used non-tunneled catheters, bloodstream infection developed in $62 \%$.

Of the patient group, $54.2 \%$ were female and $44 \%$ were older than 60 years. All patients were adults, and their ages ranged 
from 23-85 years (median, 58 years). The inpatients comprised $96.7 \%$ of the study population. Twelve $(20 \%)$ patients died during the study period.

The demographic and clinical characteristics of the patients are shown in Table 1. Bivariate analysis indicated that the BSI frequency was 1.7 times higher in patients with hypoalbuminemia $(76.5 \%)$ compared with patients with normal albumin levels. The main signs and symptoms observed in patients with BSI were chills, 35 (94.6\%); shivering, 34 (91.9\%); and hyperemia at the time of CVC insertion, 24 (64.9\%).

In total, $114 \mathrm{CVCs}$ were inserted during the study period, and $111(97.4 \%)$ of these were non-tunneled devices. The catheters were inserted into the right jugular vein in $45 \%$ of the catheterizations. None of the variables related to CVC use were associated with the development of BSI (Table 2).
With regard to changes in or evolution of vascular access, 31 patients were switched from non-tunneled to arteriovenous catheters, and 16 patients progressed to tunneled catheters.

Thirty-seven patients developed BSI, and $19(50 \%)$ of these patients were catheterized because they lacked an AVF that could be used. In the other patients $(50 \%)$, the need for urgent hemodialysis required catheterization.

Multivariate analysis revealed no association between BSI in hemodialysis patients and the following variables: hypoalbuminemia ( $\mathrm{p}=0.220 ; 95 \%$ CI $0.75<\mathrm{RR}<3.44$ ), gender ( $p=0.368 ; 95 \%$ CI $0.69<\mathrm{RR}<2.69$ ), indication for catheterization (loss, maturation, or awaiting AVF) $(p=0.670$; $95 \%$ CI $0.58<\mathrm{RR}<2.35)$, number of catheter sites $(\mathrm{p}=0.690$; $95 \%$ CI $0.54<\mathrm{RR}<2.54$ ), length of catheterization (days) $(\mathrm{p}=0.701 ; 95 \%$ CI $0.99<\mathrm{RR}<1.01)$, and number of hemodialysis sessions ( $p=0.924 ; 95 \%$ CI $0.96<R R<1.04$ ).

TABLE 1 - Number and percentage of hemodialysis patients according to study variables and occurrence of bloodstream infection.

\begin{tabular}{|c|c|c|c|c|c|c|}
\hline \multirow[b]{4}{*}{ Variables } & \multicolumn{4}{|c|}{ Bloodstream infection } & \multirow{4}{*}{$\begin{array}{c}\mathrm{RR} \\
(95 \% \mathrm{CI})\end{array}$} & \multirow[b]{4}{*}{$\mathrm{p}$} \\
\hline & \multirow{2}{*}{\multicolumn{2}{|c|}{$\begin{array}{c}\text { yes } \\
(\mathrm{n}=37)\end{array}$}} & \multirow{2}{*}{\multicolumn{2}{|c|}{$\begin{array}{c}\text { no } \\
(\mathrm{n}=22)\end{array}$}} & & \\
\hline & & & & & & \\
\hline & $\mathrm{n}$ & $\%$ & $\mathrm{n}$ & $\%$ & & \\
\hline \multicolumn{7}{|l|}{ Gender } \\
\hline male & 20 & 74.1 & 7 & 25.9 & 1 & $\mathrm{~b}_{0,097}$ \\
\hline female & 17 & 53.1 & 15 & 46.9 & $1.39(0.94-2.07)$ & \\
\hline \multicolumn{7}{|l|}{ Age (years) } \\
\hline $23-40$ & 7 & 63.6 & 4 & 36.4 & 1 & $\mathrm{c}_{0,831}$ \\
\hline $41-60$ & 13 & 59.1 & 9 & 40.9 & $1.08(0.61-1.90)$ & \\
\hline $61-85$ & 17 & 65.4 & 9 & 34.6 & $0.97(0.57-1.65)$ & \\
\hline \multicolumn{7}{|l|}{ Diabetes } \\
\hline yes & 15 & 57.7 & 11 & 42.3 & 1 & $\mathrm{~b}_{0,479}$ \\
\hline no & 22 & 66.7 & 11 & 33.3 & $0.87(0.58-1.30)$ & \\
\hline \multicolumn{7}{|c|}{ Hypoalbuminemia } \\
\hline yes & 26 & 76.5 & 8 & 23.5 & 1 & $\mathrm{~b}_{0,011}$ \\
\hline no & 11 & 44.0 & 14 & 56.0 & $1.74(1.08-2.81)$ & \\
\hline \multicolumn{7}{|l|}{ Recent surgery } \\
\hline yes & 18 & 62.1 & 11 & 37.9 & 1 & $\mathrm{~b}_{0,920}$ \\
\hline no & 19 & 63.3 & 11 & 36.7 & $0.98(0.66-1.45)$ & \\
\hline \multicolumn{7}{|c|}{ Use of corticosteroids } \\
\hline yes & 3 & 75.0 & 1 & 25.0 & 1 & $\mathrm{~d}_{1,000}$ \\
\hline no & 34 & 61.8 & 21 & 38.2 & $1.21(0.66-2.22)$ & \\
\hline \multicolumn{7}{|c|}{ Number of hemodialysis sessions } \\
\hline$<25$ & 11 & 55.0 & 9 & 45.0 & $1.14(0.69-1.88)$ & \\
\hline from 25 to 35 & 11 & 73.3 & 4 & 26.7 & $0.85(0.55-1.32)$ & \\
\hline$>35$ & 15 & 62.5 & 9 & 37.5 & 1 & $\mathrm{c}_{0,641}$ \\
\hline
\end{tabular}

Note: If $\mathrm{p} \leq 0.05$, differences are statistically significant. RR: relative risk; ${ }^{b}$ Chi-square test; ${ }^{\text {c }}$ Chi-square test for trend; ${ }^{d}$ Fisher's exact test. 
TABLE 2 - Number and percentage of patients undergoing hemodialysis according to catheter use and occurrence of bloodstream infection.

\begin{tabular}{|c|c|c|c|c|c|c|}
\hline \multirow[b]{4}{*}{ Catheter } & \multicolumn{4}{|c|}{ Bloodstream infection } & \multirow{4}{*}{$\begin{array}{c}\mathrm{RR} \\
(95 \% \mathrm{CI})\end{array}$} & \multirow[b]{4}{*}{$\mathrm{p}$} \\
\hline & \multirow{2}{*}{\multicolumn{2}{|c|}{$\begin{array}{c}\text { yes } \\
(n=37)\end{array}$}} & \multirow{2}{*}{\multicolumn{2}{|c|}{$\begin{array}{c}\text { no } \\
(\mathrm{n}=22)\end{array}$}} & & \\
\hline & & & & & & \\
\hline & $\mathrm{n}$ & $\%$ & $\mathrm{n}$ & $\%$ & & \\
\hline \multicolumn{7}{|l|}{ Indication } \\
\hline \multicolumn{7}{|c|}{ Difficulties in catheter insertion } \\
\hline yes & 24 & 66.7 & 12 & 33.3 & 1 & $\mathrm{c}_{0,432}$ \\
\hline no & 13 & 56.5 & 10 & 43.5 & $1.18(0.77-1.81)$ & \\
\hline one & 13 & 52.0 & 12 & 48.0 & $1.54(0.98-2.42)$ & \\
\hline \multicolumn{7}{|c|}{ Length of catheterization (days) } \\
\hline$\leq 50$ & 14 & 60.9 & 9 & 39.1 & $1.19(0.73-1.95)$ & \\
\hline 51 to 100 & 15 & 60.0 & 10 & 40.0 & $1.21(0.75-1.97)$ & \\
\hline$>100$ & 8 & 72.7 & 3 & 27.3 & 1 & $\mathrm{~d}_{0,574}$ \\
\hline
\end{tabular}

Note: If $\mathrm{p} \leq 0.05$, differences are statistically significant. When present, the category no information was not included in the test calculations. RR: relative risk; ${ }^{b}$ Loss, maturation, or awaiting arteriovenous fistula; ${ }^{c}$ Chi-square test; ${ }^{\mathrm{d}}$ Chi-square test for trend.

Table 3 lists the microorganisms isolated from blood and catheter-tip cultures. Of the 124 blood cultures, 65 (52.4\%) tested positive. Gram-positive cocci $(n=18 ; 27.7 \%)$ were the most common microorganism observed, and Staphylococcus aureus accounted for most of the gram-positive infections. Among the Gram-negative bacteria isolated $(\mathrm{n}=28 ; 43.1 \%)$, Stenotrophomonas maltophilia was the most prevalent. Six Candida species were isolated, the most prevalent of which were Candida parapsilosis complex species.

In 10 patients, the same type of microorganism was isolated from the blood and catheter-tip cultures. Among these cultures, the most frequently isolated pathogens were S. aureus (6) and the Burkholderia cepacia complex (4). There was no significant difference between the prior use of antibiotics and multidrug resistance of microorganisms $(\mathrm{p}=0.751 ; \mathrm{RR}=0.80 ; 95 \%$ CI, 0.26 to 2.48 ).

Of the $15 \mathrm{~S}$. aureus isolates observed in the blood and catheter-tip cultures, only one was resistant to methicillin. Seven out of nine coagulase-negative Staphylococcus (CNS) isolates were resistant to this antibiotic. All S. epidermidis $(\mathrm{n}=7)$ isolates were resistant to methicillin. Of the 14 enterobacteria isolated, none produced beta-lactamase, and one isolate of Enterobacter cloacae was resistant to ertapenem. One of the two Pseudomonas aeruginosa isolates was resistant to ceftazidime, cefepime, imipenem, meropenem, ciprofloxacin, and piperacillin-tazobactam. Acinetobacter baumannii (3) and Chryseobacterium indologenes (5) were among the most resistant Gram-negative bacteria.

\section{DISCUSSION}

The prevention and control of bacterial and fungal infections in patients with ESRD receiving hemodialysis via CVCs is a constant concern for health professionals. Although CVCs are an important component in the management of patients with ESRD, these catheters also significantly contribute to BSIs ${ }^{10}$.

In the present study, most patients were older than 58 years, a finding that corroborates those of Sesso et al. ${ }^{2}$ A previous study ${ }^{16}$ conducted in a teaching hospital in Campo Grande indicated that most patients admitted to medical wards with CVC-related complications were older than 60 years. Patients with advanced age tend to be immunologically impaired, and they often have co-existing chronic diseases, such as systemic hypertension and diabetes. We also observed co-existing chronic disease in our patients, and our findings corroborate those of other researchers ${ }^{17}$.

To our knowledge, this is the first study in central-western Brazil to describe BSIs in patients with ESRD receiving hemodialysis. The prevalence of BSIs in our group of patients was much higher $(62.8 \%)$ than that among patients attending a hemodialysis center in Pakistan $(25 \%)^{18}$.

A study by Brito et al. ${ }^{19}$ on nosocomial infection in the neonatal intensive care unit of the Uberlândia University Hospital, in Brazil, demonstrated that BSI was the main cause of nosocomial infection (69.3\%) and indicated that CVC use was an independent risk factor $(\mathrm{p}<0.05)$ for nosocomial infection. 
TABLE 3 - Microorganisms responsible for bloodstream infection in hemodialysis patients.

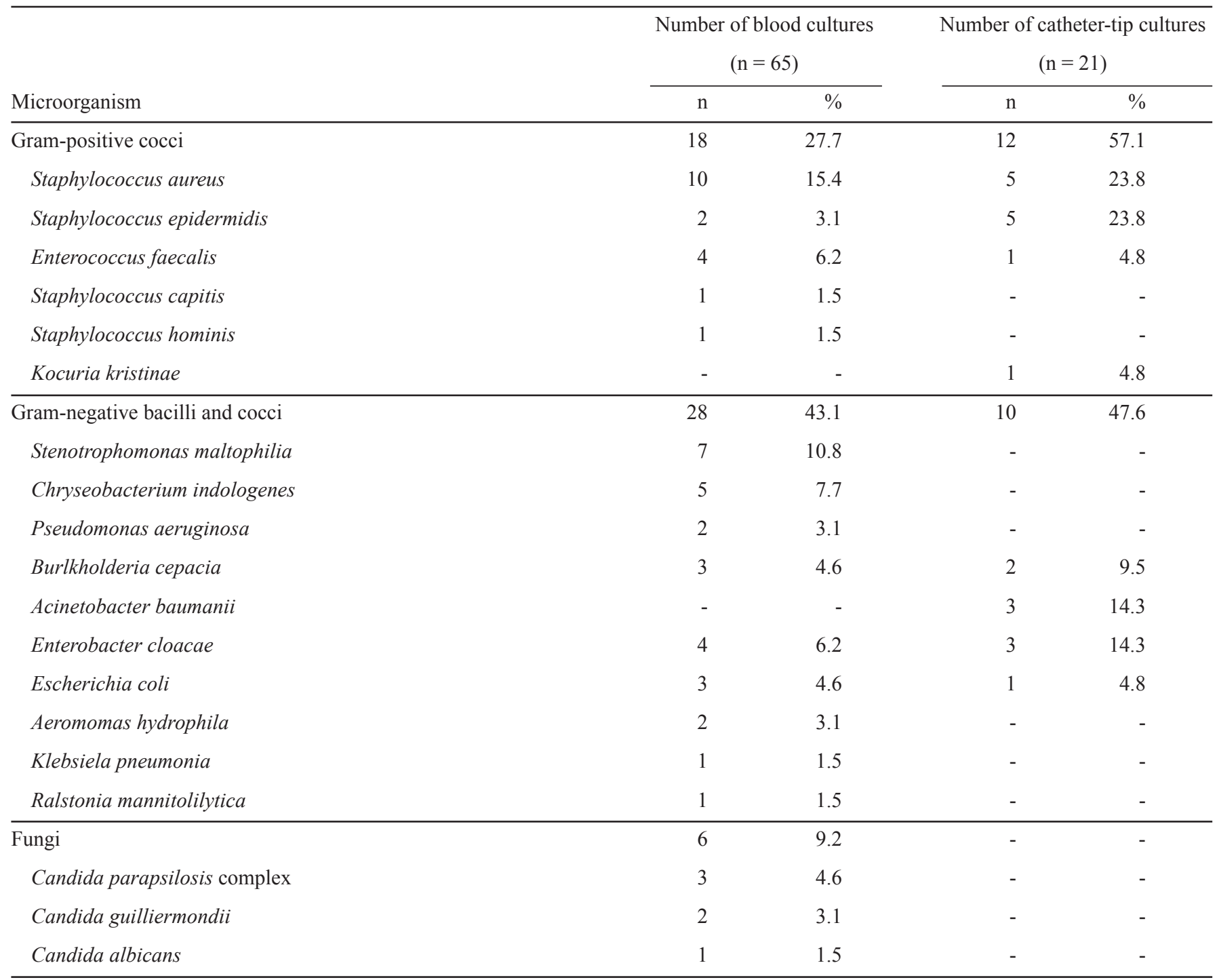

Even though previous studies ${ }^{9,18}$ reported that prolonged use of CVCs increases the risk of BSI, we observed no evidence to support this claim. In the present study, no association was observed between the long-term use of CVCs and the increased occurrence of BSI. This finding is possibly related to the small number of patients evaluated, methodological difficulties inherent in prospective studies, and specific features of the cohort (type of patients and restricted sample group).

Bivariate analysis revealed that hypoalbuminemia was a significant risk factor for BSI in our patient group. It is well known that hypoalbuminemia is associated with disease severity. Lukowsky et al. ${ }^{4}$ observed that during the first 90 days of hemodialysis, one-third of all patient deaths were associated with albumin levels of less than $3.5 \mathrm{~g} / \mathrm{dL}$. Thus, the identification and treatment of hypoalbuminemia may decrease the risk of infection.

Our results indicated a higher incidence of chills and shivering in patients who developed BSI, which was expected because these symptoms were criteria for blood culture collection. Hyperemia at the site of catheter insertion was suggestive of BSI $(\mathrm{p}<0.05)$, corroborating the results of a previous study ${ }^{20}$.

In the present investigation, non-tunneled catheters were the most frequently used catheters. Our results suggest that these catheters should be avoided in patients with ESRD due to the associated high risk of infection. We recommend that temporary catheters be replaced as soon as possible with AVFs or arteriovenous grafts. A tunneled CVC should be implanted if this is not possible ${ }^{18,21}$.

In our study, the right jugular vein was the most common access route for CVC. This finding is similar to the findings of Jones et al..$^{22}$ The jugular vein is the preferred access point for CVCs, as subclavian vein stenosis can be avoided and because the site allows for future preparation of $\mathrm{AVFs}^{21}$. Nevertheless, Grothe et al. ${ }^{23}$ reported that patients receiving hemodialysis who had CVCs inserted in the jugular vein were 56\% more likely to develop BSI than those who had catheters implanted in the subclavian vein. 
We also observed that a large percentage of our patients $(\mathrm{n}=35 ; 59.3 \%)$ began hemodialysis due to an emergency situation and therefore required CVCs. This finding is similar to the results of other studies ${ }^{24,25}$. We believe that these emergency cases were related to delayed diagnosis of ESRD.

Although blood culture tests are considered the gold standard in the diagnosis of bacteremia and fungemia, previous studies reported variable sensitivity of these tests in detecting these infections and low sensitivity in identifying patients treated with antimicrobials ${ }^{11,26}$. The high (52.4\%) percentage of positive blood cultures in our study may be a result of the nature of the population investigated. Patients receiving hemodialysis are most often individuals who present with concomitant chronic illnesses, and they are prone to immunodeficiency.

Similar to the findings of Bevilacqua et al. ${ }^{27}$, we observed that Gram-negative bacteria were predominant among the isolates. The infections caused by non-fermenting Gram-negative bacilli, such as Acinetobacter baumannii, Pseudomonas aeruginosa, Chryseobacterium indologenes, and Stenotrophomonas maltophilia, are difficult to treat because they are highly resistant, can cause outbreaks of nosocomial infection, and are often associated with high mortality rates ${ }^{28,29}$.

In this study, Staphylococcus aureus was the most frequent pathogen observed in blood cultures. Classically, these pathogens are often associated with BSIs because they are part of the skin microbiota. To date, numerous reports have indicated that BSIs associated with Staphylococcus aureus are serious and can result in severe metastatic infections such as sepsis, endocarditis, and endophthalmitis ${ }^{30,31}$.

BSIs caused by $S$. aureus require aggressive treatment, including catheter removal. In addition, if the infection does not resolve within $72 \mathrm{~h}^{30}$, transesophageal echocardiography should be performed to rule out other potential sources of infection.

The emergence of multidrug-resistant gram-positive pathogens, including methicillin-resistant Staphylococcus aureus (MRSA), coagulase-negative Staphylococcus (MRSCN), and vancomycin-resistant Enterococcus spp. (VRE), complicates the management of infections caused by these agents ${ }^{32}$.

The isolation of Kocuria kristinae in the catheter-tip cultures is a rare finding. Dunn et al. ${ }^{33}$, previously described a case of severe intravascular infection complicated by septic pulmonary embolus and thrombosis in a patient with hyperemesis gravidarum as a result of this pathogen and the use of parenteral nutrition by a CVC.

Candida yeasts, which are recognized as opportunistic pathogens, gain access to the bloodstream due to skin breakdown and are predominantly found in immunodeficient patients ${ }^{34}$. Non-albicans Candida species, such as Candida parapsilosis, have emerged as important infectious agents ${ }^{35}$. This pathogen can secrete glycosylated proteins that form biofilms in solution and adhere to plastic materials such as intravascular catheters ${ }^{36}$. Brito et al ${ }^{37}$ reported that Candida parapsilosis candidemia is associated with neutropenia, the use of CVCs, and chemotherapy.

Two patients had positive blood cultures for Candida guilliermondii for long periods, namely 6 and 12 months.
Because of the clinical condition of these patients and the difficulty of establishing new venous access, nephrologists chose not to remove the catheters. Based on a previous study ${ }^{35}$, C. guilliermondii can be considered an emerging candidemia pathogen in central-western Brazil.

We propose that in a hemodialysis unit a multidisciplinary team should conduct surveillance studies to detect BSIs. To this end, the necessary measures to prevent bacteremia and other complications associated with infection in patients with ESRD can be instituted in a timely manner. Since our study was conducted, one of the measures instituted to reduce the rate of BSI in patients with ESRD is the use of connectors in closed access systems and the exchange of these connectors every seven days. Other measures should also be implemented, such as the training of multidisciplinary teams to specifically take care of CVCs in patients with ESRD. The use of antibiotic seals and thrombolytic agents is also recommended.

In conclusion, it is important to identify which pathogens cause BSIs among patients in hemodialysis units because this information will guide initial therapy ${ }^{27}$. Surveillance studies by multidisciplinary teams are necessary so that appropriate measures can be implemented to prevent bacteremia and other complications associated with infection in patients with ESRD. We observed a high prevalence of Gram-negative, bacteriarelated BSIs among our patients with ESRD who received hemodialysis via a CVC.

\section{CONFLICT OF INTEREST}

The authors declare that there is no conflict of interest.

\section{REFERENCES}

1. Eckardt KU, Bärthlein B, Baid-Agrawal S, Beck A, Busch M, Eitner F, et al. The German Chronic Kidney Disease (GCKD) study: design and methods. Nephrol Dial Transplant 2012; 27:1454-1460.

2. Sesso RC, Lopes AA, Thomé FS, Lugon JR, Santos DR. Relatório do censo brasileiro de diálise de 2010. J Bras Nefrol 2011; 33:442-447.

3. Xue Hui, Ix JH, Wang W, Brunelli SM, Lazarus M, Hakim R, et al. Hemodialysis Access Usage Patterns in the Incident Dialysis Year and Associated Catheter-Related Complications Am J Kidney Dis 2012; 61:123-130.

4. Lukowsky LR, Kheifets L, Arah OA, Nissenson AR, Kalantar-Zadeh K. Patterns and predictors of early mortality in incident hemodialysis patients: new insights. Am J Nephrol 2012; 35:548-558.

5. Moysés Neto M, Vieira-Neto OM, Figueiredo JFC. Bacterial colonization in a hemodialysis dual lumen temporary catheter. Rev Soc Bras Med Trop 2003; 36:431-432.

6. Labeau SO, Vandijck DM, Rello J, Adam S, Rosa A, Wenisch C, et al. EVIDENCE Study Investigators. Centers for Disease Control and Prevention guidelines for preventing central venous catheter-related infection: results of a knowledge test among 3405 European intensive care nurses. Crit Care Med 2009; 37:320-323.

7. Ministério da Saúde. Agência Nacional de Vigilância Sanitária. Orientações para prevenção de infecção primária de corrente sanguínea. Brasília: Ministério da Sáude; 2010.

8. Lewis SS, Sexton DJ. Metastatic Complications of Bloodstream Infections in Hemodialysis Patients. Semin Dial 2013; 26:47-53. 
9. Bicudo D, Batista R, Furtado GH, Sola A, Medeiros EAS. Risk factors for catheter-related bloodstream infection: a prospective multicenter study in Brazilian intensive care units. Braz J Infect Dis 2011; 15:328-331.

10. Bonfante GMS, Gomes IC, Andrade ELG, Lima EM, Acurcio FA, Cherchiglia ML. Duration of temporary catheter use for hemodialysis: an observational, prospective, evaluation of renal units in Brazil. BMC Nephrol 2011; 12:1-7.

11. Ministério da Saúde. Agência Nacional de Vigilância Sanitária. Critérios nacionais de diagnósticos de infecções relacionadas a assistência a saúde- Corrente Sanguínea, Brasília: Ministério da Saúde; 2009 [Cited 2013 January 23]. Available at: http://www.anvisa.gov.br/servicosaude/ manuais/index.htm

12. Wang I-Kuan, Chang Yi-Chin, Liang Chih-Chia, Chuang Feng-Rong, Chang Chiz-Tzung, Lin Hsin-Hung, et al. Bacteremia in Hemodialysis and Peritoneal Dialysis Patients. Intern Med 2012; 51:1015-1021.

13. Mermel LA, Allon M, Bouza E, Craven DE, Flynn P, O' Grady NP, et al. Clinical practice guidelines for the diagnosis and management of intravascular catheter-related infection: 2009 Update by the Infectious Diseases Society of America. Clin Infect Dis 2009; 49:1-45.

14. Ayres M, Ayres Jr M, Ayres DL, Santos AAS. BioEstat [Software]. Aplicações estatísticas das Ciências Bio-médicas [programa de computador]. Versão 5.0. Belém (PA): Sociedade Mamirauá; 2007.

15. Centers for Disease Control and Prevention (CDC). Division of Public Health Surveillance and Informatics. Epi Info [Software], [Cited 2011 February 01] Version 3.5.3, 26 January 2011. Available at http://www. cdc.gov/.

16. Loureiro MDR, Barbosa DA. Risco de infecção hospitalar em pacientes submetidos a cateterização venosa central. In: Universidade Federal do Mato Grosso do Sul, editor. Dimensões do processo de cuidar em enfermagem. Campo Grande: Editora UFMS. 2004, p. 341-353.

17. Barros MB, Francisco PM, Zanchetta LM, César CL. Trends in social and demographic inequalities in the prevalence of chronic diseases in Brazil. PNAD: 2003-2008. Cien Saude Colet 2011; 16:3755-3768.

18. Qureshi AL, Abid K. Frequency of catheter related infections in haemodialysed uraemic patients. J Pak Med Assoc 2010; 60:671-675.

19. Brito DVD, Brito CS, Resende DS, Moreira do Ó J, Abdallah VOS, G Filho PP. Nosocomial infections in a Brazilian neonatal intensive care unit: a 4 year surveillance study. Rev Inst Med Trop S Paulo 2010; 43:633-637.

20. Fermi MRV. Diálise para enfermagem: Guia prático. $2^{\text {nd }}$ ed. Rio de Janeiro: Guanabara Koogan; 2010.

21. Vanholder R, Canaud B, Fluck R, Jadoul M, Labriola L, Marti-Monros $\mathrm{A}$, et al. Diagnosis, prevention and treatment of haemodialysis catheterrelated bloodstream infections (CRBSI): a position statement of European Renal Best Practice (ERBP). Nephr Dial Trans Plus 2010; 3:234-246.

22. Jones SM, Ravani P, Hemmelgarn BR, Muruve D, Macrae JM. Morphometric and biological characterization of biofilm in tunneled hemodialysis catheters. Am J Kidney Dis 2011; 57:449-455.
23. Grothe C, Belasco AGS, Bittencourt ARC, Vianna LAC, Sesso RCC, Barbosa DA. Incidência de infecção da corrente sanguínea nos pacientes submetidos à hemodiálise por cateter venoso central. Rev Latino-Am Enfermagem 2010; 18:8.

24. Souza RA, Oliveira EA, Silva JM, Lima EM. Avaliação do acesso vascular para hemodiálise em crianças e adolescentes: um estudo de coorte retrospectivo de 10 anos. J Bras Nefrol 2011; 33:422-430.

25. Ethier J, Mendelssohn DC, Elder SJ, Hasegawa T, Akizawa T, Akiba $\mathrm{T}$ et al. Vascular access use and outcomes: an international perspective from the Dialysis Outcomes and Practice Patterns Study. Nephrol Dial Transplant 2008; 23:3219-3226.

26. Huttunen R, Aittoniemi J. New concepts in the pathogenesis, diagnosis and treatment of bacteremia and sepsis. J Infect 2011; 63:407-419.

27. Bevilacqua JL, Gomes JG, Santos VF, Canziani, ME. Comparação ente citrato trissódico e heparina como solução para selo de cateter em pacientes em hemodiálise. J Bras Nefrol 2011; 33:86-92.

28. Al Mohajer M, Darouiche RO. Sepsis syndrome, bloodstream infections and device-related infections. Med Clin N Am 2012; 96:1203-1223

29. Souza MMRS, Silva CAB, Paschoalin EL, Moura Júnior JAM, Paschoalin RP, Oliveira EP. Bacteriemia por Chryseobacterium indologenes em diabético em hemodiálise ambulatorial. J Bras Patol Med Lab 2012; 48:29-31.

30. Fitzgibbons LN, Puls DL, Mackay K, Forrest GN. Management of Grampositive coccal bacteremia and hemodialysis. Am J Kidney Dis 2011; 57:624-640

31. Troidle L, Finkelstein FO. Catheter-related bacteremia in hemodialysis patients: the role of the central venous catheter in prevention and therapy. Int J Artif Organs 2008; 31:827-833.

32. Ager S, Gould K. Clinical update on linezolid in the treatment of Grampositive bacterial infection. Infect and drug resistance 2012; 5: 87-102.

33. Dunn R, Bares S, David MZ. Central venous catheter-related bacteremia caused by Kocuria kristinae: case report and review of the literature. Ann Clin Microb Antimicrob 2011; 10:2-5.

34. Xavier PCN, Chang MR, Nunes MO, Palhares DB, Silva RA, Bonfim GF, Junior NFA. Neonatal candidemia in a public hospital in Mato Grosso do Sul. Rev Soc Bras Med Trop 2008; 41:459-463.

35. Chang MR, Correia FP, Costa LC, Xavier PCN, Palhares DB, Taira DL, et al. Candida Bloodstream infection: data from a teaching hospital in Mato Grosso do Sul, Brazil. Rev Inst Med Trop S Paulo 2008; 50:265-268.

36. Colombo AL, Nucci M, Park Bj, Nouér SA, Arthington-Skaggs B, Matta DA, et al. Epidemiology of candidemia in Brazil: a nationwide sentinel surveillance of candidemia in eleven medical centers. J Clin Microbiol 2006; 44:2816-2823.

37. Brito LR, Guimarães T, Nucci M, Rosas RC, Almeida LP, Matta DA, et al. Clinical and microbiological aspects of candidemia due to Candida parapsilosis in Brazilian tertiary care hospitals. Med Mycol 2006; 44:261-266 